\title{
Elementary Modules in Games Networks
}

\author{
Matthieu Manceny and Franck Delaplace \\ IBISC, FRE 2873 CNRS - University of Evry \\ 523 Place des Terrasses, 91000 Evry, France
}

\begin{abstract}
In this paper we propose an original modular extension of game theory named games network. The objective of games networks is to provide a theoretical framework which suits to modular dynamics resulting from different local interactions between various agents and which enables us to describe complex system in a modular way. Games networks describes situations where an agent can be involved in several different games, with several different other agents, at the same time. In particular, we focus on the determination of global equilibria, resulting from the composition of local equilibria for each game of the network.

However, several games networks can represent the same dynamics. We define the notion of dependence between agents, which allows us to compute a games network normal form. This normal form emphasizes the elementary modules which compose the games network.
\end{abstract}

Keywords: complex systems, modularity, game theory, networks, dynamics.

\section{Introduction}

Analysis of complex systems is often based on the studies of relationships between components instead of elements themselves. This puts the emphasis on the way to analyze interactions. From modeling standpoints, networks provide a suitable framework to describe interactions (edges) of components (vertices). With networks, the description remains static and it is mainly focused on the structural analysis of the properties of the system. For example, a network where the connectivity degree follows a power law identifies robustness properties to the "not targeted" attacks $([1,3,6])$. Thus, the study of interacting networks within a modeling framework is based on a parallel between structural properties of the network (e.g. power law) and dynamical properties (e.g. robustness).

In order to improve the framework by including dynamical aspects for the analysis of interactions, we propose to mix two formalisms: network formalism and game theory.

Game theory has been pioneered by von Neumann and Morgenstern to define a theoretical framework to model complex interactions between agents. It studies how the interacting agents (or players) make their choices (or strategies) evolved considering their interactions with other players (13]). Applications of game theory are larger than "Games", and characterize complex interactions in fields such as Biology $([8,11])$, Economy $([7,9])$ or Computer Science $([2,12])$. The 
choices players make characterize the dynamical aspects of game theory. The notion of Nash equilibrium captures the steady states of a game.

Theory of games networks is an extension of game theory where a player can be involved in several games simultaneously. Games networks can be viewed as a "network of games and players" where players are connected to the games they participate to. With games networks, we describe the interactions as a set of modular activities where each game represents a module of interactions. We can study how the dynamics of one game, defined by local equilibria, influences the dynamics of the whole network, defined by global equilibria.

In order to analyze and understand interactions between components of a network, we search for the elementary interactions within this network. We define a separation algorithm which decomposes one game in its elementary modules underlining elementary interactions. From their reduced size, elementary modules should be more comprehensible than the games of the starting network. Moreover, elementary modules identify structures which would be impossible to characterize if agents are separately considered.

The paper is organized as follow: section 2 presents fundamental notions of game theory and theory of games networks; section 3 deals with research of elementary modules and describes the separation algorithm. We conclude in section 4 .

\section{Theory of Games Networks}

In this section we present an original extension of game theory: the theory of games networks. In order to ease the reading of the paper, we first briefly recall notions of game theory; then we introduce the extension. The reader may refer to [10] or [5] for a more complete presentation.

\subsection{Game Theory}

Strategic Games. Strategic game theory proposes a model of interactions where interacting agents, the players, choose their action, their strategy, once and for all and simultaneously. Moreover, each player is rational — it aims at maximizing its payoff — and perfectly informed of other players' payoffs. The formal definition of a strategic game is as follow:

Definition 1 (Strategic game). A strategic game is a triplet $\langle A, C, u\rangle$ where:

- $A$ is the set of players.

$-C=\left\{C_{i}\right\}_{i \in A}$ is a set of strategy sets; $C_{i}=\left\{c_{i}^{1}, \ldots, c_{i}^{m_{i}}\right\}$ is the set of player $i$ 's strategies.

$-u=\left(u_{i}\right)_{i \in A}$ is the payoff vector; $u_{i}: \times_{i \in A} C_{i} \mapsto \mathbb{R}$ is a function which maps a payoff for player $i$ considering a game configuration, i.e. other players' strategies. 


Payoff table
\begin{tabular}{|r|rr|}
\hline$x / y$ & Off & On \\
\hline Off & $(1,1)$ & $(0,2)$ \\
On & $(3,0)$ & $(-1,-1)$ \\
\hline
\end{tabular}

Nash equilibria

$\{(\mathrm{On}, \mathrm{Off}),(\mathrm{Off}, \mathrm{On})\}$

Fig. 1. Example of a $2 \times 2$ strategic game

Representation by Table. $2 \times 2$ strategic games -2 players with 2 strategies are usually used to present game theory notions. Such a game can be represented by a table where first player's strategies are in line, and second player's ones in column. Considering example from fig. 1 if player $x$ play its Off strategy $y$ its On strategy, then the payoff attributed to $x$ is 0 and 2 for player $y$.

Nash Equilibrium. Nash equilibrium is a central concept which captures the steady configurations of a strategic game:

Definition 2 (Nash equilibrium). Let $\left\langle A, C=\left\{C_{i}\right\}_{i \in A}, u=\left(u_{i}\right)_{i \in A}\right\rangle$ be a strategic game. A Nash equilibrium is a game configuration $c^{*} \in \times_{i \in A} C_{i}$ such that:

$$
\forall i \in A, \forall c_{i} \in C_{i}, u_{i}\left(\left(c_{-i}^{*}, c_{i}\right)\right) \leq u_{i}\left(c^{*}\right)
$$

with $\left(c_{-i}^{*}, c_{i}\right)$ equivalent to the game configuration $c^{*}$ but where player $i$ plays its strategy $c_{i}$ rather than $c_{i}^{*}$.

In a Nash equilibrium, the strategy played by player $i$ is the best possible response to strategies played by other players. In other words, no agent can unilaterally deviate from a Nash equilibrium without decreasing its payoff. Considering example from fig. 1, 2 Nash equilibria exist: $(x=0 \mathrm{ff}, y=0 \mathrm{n})$ and $(x=0 \mathrm{n}, y=0 \mathrm{ff})$.

\subsection{Games Networks}

Strategic Games Networks. In game theory, all the agents are interacting together. Theory of games networks is an original framework which extends game theory and allows a modular description of the network dynamics. Thus, players can participate to several games simultaneously. Games which composed the games network can be seen as dynamical modules which describe the local interactions between agents. The formal definition of a strategic games network is as follow:

Definition 3 (Games network). A strategic games network is a triplet $\langle\mathcal{A}, C, \mathcal{U}\rangle$ where:

- $\mathcal{A}$ is the set of players.

$-C=\left\{C_{i}\right\}_{i \in A}$ is a set of strategy sets; $C_{i}=\left\{c_{i}^{1}, \ldots, c_{i}^{m_{i}}\right\}$ is the set of player $i$ 's strategies.

$-\mathcal{U}=\left\{\left\langle A_{j}, u^{j}\right\rangle\right\}$ is a set of games. For each game, $A_{j} \subseteq \mathcal{A}$ is the set of players and $u^{j}=\left(u_{i}^{j}: \times_{i \in A_{j}} C_{i} \mapsto \mathbb{R}\right)_{i \in A_{j}}$ is the payoff vector. 


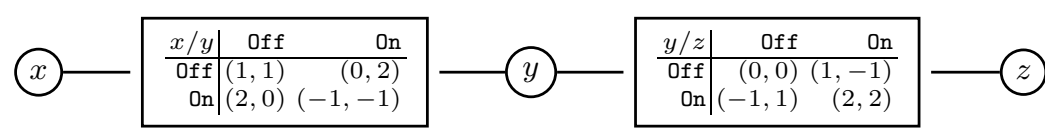

Global equilibria: $\{(x=0 \mathrm{n}, y=0 \mathrm{ff}, z=0 \mathrm{ff}),(x=0 \mathrm{ff}, y=0 \mathrm{n}, z=0 \mathrm{n})\}$

Fig. 2. Example of a 3-players-2-games games network

Given a game, it is not necessary to recall the strategies available for an agent because they are identical for all the game this agent participates to. Thus, strategies are associated to the agent rather than to each game.

Graphical Representation. Games networks are represented by bipartite graphs (fig. 2). In such a graph, players are represented by a circle and games by rectangles. Players are connected to games they participate to.

Equilibria. Two types of dynamics emerge from the games network representation: the first one is local to each game and the second one is global to the whole network. Thus, two notions of equilibria have been defined: local equilibria and global equilibria. Local equilibria correspond to Nash equilibria for each game which composed the network. Considering example from fig. 2] two local equilibria exist for the $x / y$ game $((x=0 \mathrm{ff}, y=\mathrm{On})$ and $(x=0 \mathrm{n}, y=0 \mathrm{ff}))$ and two local equilibria for the $y / z$ game $((y=0 \mathrm{ff}, z=0 \mathrm{ff})$ and $(y=0 \mathrm{n}, z=0 \mathrm{n}))$. Global equilibria correspond to a game configuration which define local equilibria for all the games of the network. Global equilibria can be computed by a combination of local equilibria. Considering example from fig. 2. strategies available for player $y$ are $\mathrm{Off}$ or $\mathrm{On}$, which corresponds to two global equilibria, $(x=$ On, $y=0 \mathrm{ff}, z=\mathrm{Off})$ and $(x=\mathrm{Off}, y=\mathrm{On}, z=\mathrm{On})$.

\section{$3 \quad$ Elementary Modules}

\subsection{Structure and Network Equivalence}

In games networks, each game of the network is naturally identified to a module. Thus, a games network can be seen as a composition of modules linked through the agents. Each module defines a local dynamics and the network structure the way the modules are linked - defines a global dynamics. These dynamics are observed by their steady states: local and global equilibria.

However, a same dynamics can be modeled by different structures. Considering the example from fig. 3, the one-game-three-players network, on the left, has the same global equilibria, and the same dynamics, than the two-games network, on the right. We say the two networks are equivalent.

Thus, we have to search for a "normal form", that is a canonical representation of a games networks. A games network normal form is defined as an equivalent games network whose games involved as few players as possible. Games in a games network normal form are called "elementary modules". 


\subsection{Algorithm}

Algorithm from fig. 4 separates a game in elementary modules 1 . It is based on the notion of player dependence.

Dependence. Intuitively, a player $a$ depends on a player $b$ if $a$ 's payoffs are altered by $b$ 's strategies. Formally, dependence is defined as follow:

Definition 4 (Dependence). Let $\langle A, C, u\rangle$ be a strategic game. Let $j \neq i \in A^{2}$ be two agents. $j$ depends on $i$, denote by $i \delta_{u} j$, if:

$$
\exists c_{i} \in C_{i}, \exists c_{i}^{\prime} \in C_{i}, \exists c_{-i} \in C_{-i}, u_{j}\left(c_{-i}, c_{i}\right) \neq u_{j}\left(c_{-i}, c_{i}^{\prime}\right)
$$

More precisely, the algorithm refers to the notion of predecessors:

Definition 5 (Predecessors). Let $\langle A, C, u\rangle$ a strategic game. We note $\delta_{u}^{-}(j)$, $j \in A$, the set of $j$ 's predecessor:

$$
\forall j \in A, \delta_{u}^{-}(j)=\left\{i \in A \mid i \delta_{u} j \wedge i \neq j\right\}
$$

Notions of dependence and predecessors are used to underline interactions between agents. It allows us to determine which agents have to be involved in the same elementary module.

Payoffs. Once we have find players involved in an elementary module, payoffs have to be attributed. Let $a \in A$ be a player which participates to an elementary module $G$ :

- if all of $a$ 's predecessors are in $G$, we can easily compute $a$ 's payoffs, because none of the absent players has any influence on $a$ 's payoffs. The pick function in the separation algorithm chooses one configuration of the starting game where strategies for $a$ 's predecessors are identical to their strategies from the elementary module, and compute $a$ 's payoffs.

- if one of $a$ 's predecessors is not in $g$, then $a$ 's payoffs are 0 .

The separation is illustrated in fig. 3 fig. 4 details the algorithm.

\section{Conclusion}

In this paper, we presented the theory of games networks to study modularity of interacting networks. Theory of games networks extends game theory with the possibility to define local interactions between agents. These local interactions are modeled by the different games which constitute the network, and are observed through their local equilibria. At the scale of the whole network, local equilibria combine to form global equilibria. The same complex dynamics can be represented by several different games networks. We were interested in a

\footnotetext{
${ }^{1}$ For more information, please refer to $[5]$.
} 
Game (payoffs table)

\begin{tabular}{|rrr|rrr|}
\hline$x$ & $y$ & $z$ & $u^{x}$ & $u^{y}$ & $u^{z}$ \\
\hline Off & Off & Off & 1 & 2 & -1 \\
Off & Off & On & 1 & 2 & 0 \\
Off & On & Off & -1 & 0 & -1 \\
Off & On & On & -1 & 0 & 0 \\
On & Off & Off & 0 & 0 & 0 \\
On & Off & On & 0 & 0 & -1 \\
On & On & Off & 3 & 3 & 0 \\
On & On & On & 3 & 3 & -1 \\
\hline
\end{tabular}

Normal form

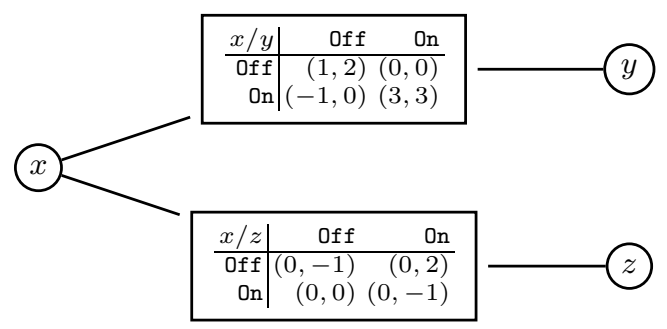

Fig. 3. A 3-players game and its normal form

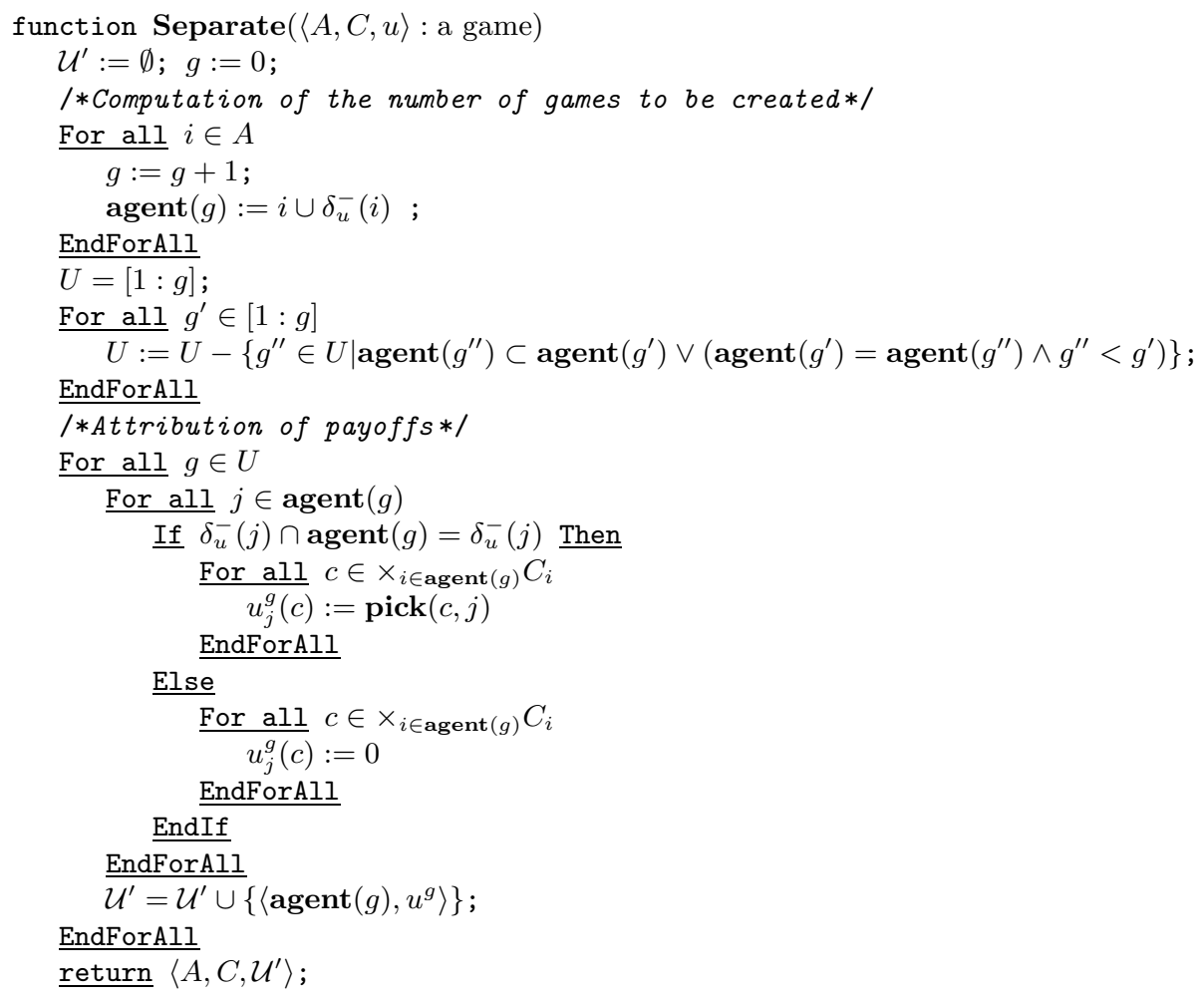

Fig. 4. Separation algorithm

canonical representation of games networks - the normal form - where each game involved as less agents as possible. In normal form, games are qualified as elementary modules and connect the "more connected" agents.

Theory of games networks has been used in a real case to model a part of the Plaminogen Activator system (PAs) which is involved in the migration of cancer cells. The games network of PAs is composed of 10 biological agents 
(such as molecules or proteins) and 6 games. The modelisation has underlined the central role of one player (the Plasminogen Activator Inhibitor, PAI-1), and the existence of two global equilibria which correspond to physiological states of the cells. These results were found in experiments. The reader may refer to [4] to know more about PAs and its games networks modelisation.

\section{References}

1. R. Albert and A.-L. Barabasi. Statistical mechanics of complex networks. Reviews of Modern Physics, 74:47-97, 2002.

2. R. Alur, T.A. Henzinger, and O. Kupferman. Alternating-time temporal logic. In Proceedings of the 38th IEEE Symposium on Foundations of Computer Science, Florida, October 1997.

3. A. Barabasi. Linked: How Everything Is Connected to Everything Else and What It Means. Plume, 2003.

4. C. Chettaoui, F. Delaplace, M. Manceny, and M. Malo. Games Network \& Application to PAs system. In Information Processing in Cells and Tissues (IPCAT), 2005 .

5. F. Delaplace and M. Manceny. Games network. Technical Report 101-2004, Laboratoire de Méthodes Informatiques (LaMI), CNRS-UMR 8042, University of Evry, 2004. http://www.lami.univ-evry.fr/ mmanceny/.

6. H. Jeong, B. Tombor, R. Albert, Z. N. Oltvai, and A. Barabasi. The large-scale organization of metabolic networks. Nature, 407:651-654, 2000.

7. D. M. Kreps. A Course in Microeconomic Theory. Princeton University Press, 1990.

8. J. Maynard Smith. Evolution and the Theory of Games. Cambridge Univ. Press, 1982.

9. R. D. McKelvey and A. McLennan. Computation of equilibria in finite games. In Handbook of Computational Economics, volume 1, pages 87-142. Elsevier, 1996. http://econweb.tamu.edu/gambit/.

10. R. B. Myerson. Game Theory: Analysis of Conflict. Harvard University Press, 1991.

11. M. A. Nowak and K. Sigmund. Evolutionary dynamics of biological games. Sciences, 303(6):793-799, februar 2004.

12. C. H. Papadimitriou. Game theory and mathematical economics: a theoretical computer scientist's introduction. In 42nd IEEE Symposium on Foundations of Computer Science: Proceedings, pages 4-8, 2001.

13. J. Von Neumann and O. Morgenstern. Theory of games and economic behavior. Princeton University Press, Princeton, New Jersey, first edition, 1944. 